\title{
Identifying and Characterizing Stellar Clusters Toward the Galactic Anti-Center from Pan-STARRS1 Survey
}

\author{
C.-C. Lin ${ }^{1,2}$, W.-P. Chen ${ }^{2}$, J. Hou ${ }^{1}$, L. Chen ${ }^{1}$ and Z. Shao ${ }^{1}$ \\ ${ }^{1}$ Shanghai Astronomical Observatory, CAS, \\ 80 Nandan Road, Shanghai 200030, China \\ email: cclin@shao.ac.cn \\ ${ }^{2}$ Graduate Institute of Astronomy, National Central University, \\ 300 Jhongda Road, Jhongli 32001, Taiwan
}

\begin{abstract}
The current sample of star clusters (SCs) is still incomplete beyond $1.8 \mathrm{kpc}$. The Pan-STARRS1 with its wide field of views and sensitive cameras provides us an opportunity to identify and characterize SCs as much as possible. By using a star counting algorithm, we obtained a total of 491 stellar density enhancements in a field of $20^{\circ} \times 20^{\circ}$ toward the Galactic Anti-Center (GAC), of which 50 are known SCs. The remaining 441 candidates are verified with radii, interstellar reddening, distances, and ages with proper motions and multiple bands photometries. Our results push the completeness of SCs almost near the edge of GAC. With the revised SC sample, the separation between Sagittarius and Perseus arms are about $3.2 \pm 0.2 \mathrm{kpc}$ and the widths of the nearby spiral arms - Sagittarius, Orion, and Perseus - with are $1.4 \pm 0.1$, $1.6 \pm 0.1$, and $3.3 \pm 0.2 \mathrm{kpc}$, respectively.
\end{abstract}

Keywords. Galaxy: structure; open clusters and associations: general

\section{Motivation and Pan-STARRS1}

Some hundreds of thousand star clusters (SCs) are expected to exist on the Galactic plane based on the number of present SCs in the solar neighborhood (Piskunov et al. 2006). However, in the past few decades, the catalogs of SCs contain only a few thousand entries and mostly limited in $1.8 \mathrm{kpc}$ (Kharchenko et al. 2013). The discrepancy is due partly to dust extinction in the Galactic plane, and partly to lack of comprehensive all-sky searches for distant systems.

Our goal is to conduct a comprehensive search for SCs from Pan-STARRS1 - the Panoramic Survey Telescope And Rapid Response System - which is equipped with a $1.8 \mathrm{~m}$ telescope, a 1.4 giga-pixel camera, and multiple optical bands to a depth of about 24 magnitude, about 100 times more wide and sensitive than currently available surveys (Kaiser et al. 2010). Here, we present the pilot study by applying a star-counting algorithm (Lin et al. 2011) to the Pan-STARRS1 data, which contains about 30 million objects in the Galactic Anti-Center (GAC) with a $400 \mathrm{deg}^{2}$ field of view.

\section{Identification and Characterization of Star Cluster Candidates}

By using a star-counting algorithm, 491 stellar density enhancements were identified toward the GAC with a $400 \mathrm{deg}^{2}$. Among them, 49 were matched with known open clusters and one was matched with a globular cluster, Palomar 2. A total of 109 known SCs were located toward the GAC. Except for larger radii, and embedded ones, the detection rate of SCs with radii smaller than $10^{\prime}$ from the algorithm used was $50 / 60 \approx 83 \%$. 

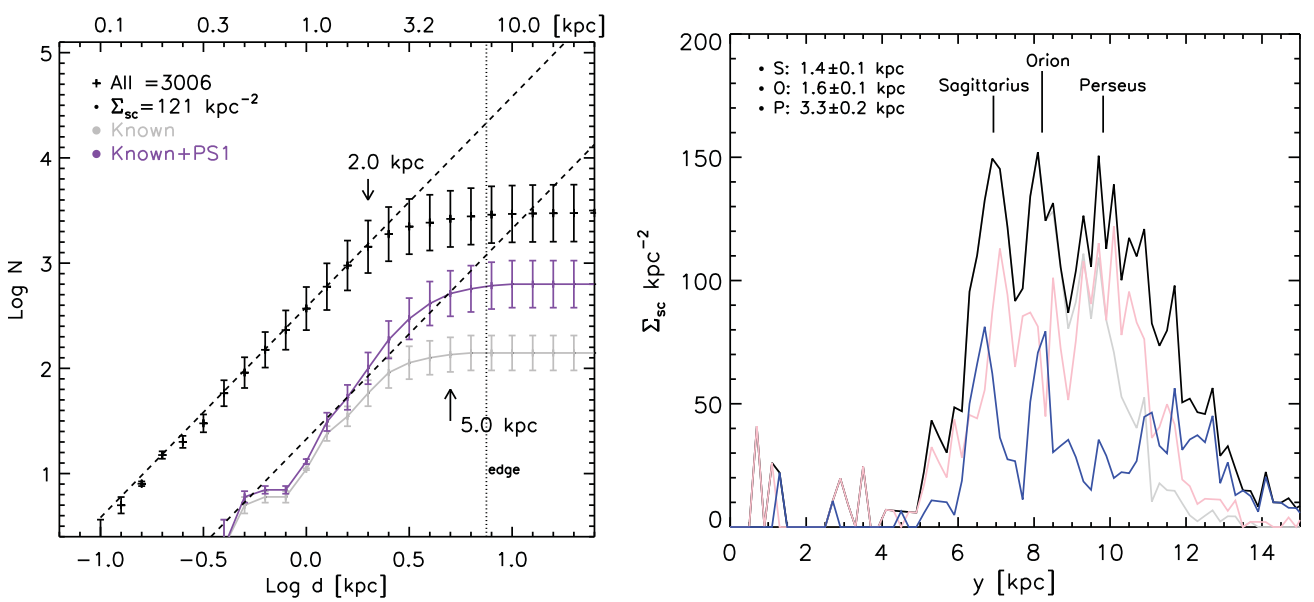

Figure 1. (Left) The cumulative number distribution as a function of the heliocentric distance of the PS1 SC candidates. The purple line shows cumulative number density distribution of 491 candidates combined with known SCs. Poisson errors are marked. (Right) The SCs surface density distribution from Galactic center to GAC. The thick grey line represents known SC sample from Kharchenko et al. (2013). The black line demonstrates the revised SC sample. The pink line shows older age of SCs than $100 \mathrm{Myr}$, while the blue line represents SCs younger than 100 Myr.

The remaining 441 candidates were characterized with radius, reddening, distance, age, and lowest mass members have been implemented on these candidate along with proper motions and multi-band photometry. Compared with known SCs, the resulting reddening values and distances are generally larger because of the optical filters are more sensitive to dust extinction. The age determination for star clusters with red giant members are more trustworthy than those without red giant members.

\section{Discussion and Future Works}

The revised SC sample is estimated to be complete up to a heliocentric distance of $5 \mathrm{kpc}$ toward the GAC, see Figure 1. Moreover, this sample allows us to estimate the separation between Sagittarius and Perseus arms to be about $3.2 \mathrm{kpc}$ with $0.2 \mathrm{kpc}$ uncertainty and the widths of the nearby spiral arms - Sagittarius, Orion, and Perseus - with the fullwidth-half-maximum to be $1.4 \pm 0.1,1.6 \pm 0.1$, and $3.3 \pm 0.2 \mathrm{kpc}$, respectively.

The candidates in the current search still need to be verified to be real SCs or not either by Pan-STARRS1 images inspection or by follow-up observations. The age, in particular, needs to be determined with not only the Padova model with different metallicity, but also the models providing pre-main sequence isochrones. The spectroscopic surveys, such as SDSSIV-APOGEE2 and LAMOST, will provide the radial velocity information for members in SCs so that the 3D motion of SCs in the Milky way can be estimated to trace how SCs interact with the spiral arms or giant molecular clouds.

\section{References}

Kaiser, N., Burgett, W., Chambers, K., et al. 2010, in SPIE, Vol. 7733

Kharchenko, N. V., Piskunov, A. E., Schilbach, E., et al. 2013, A\&A, 558, A53

Lin, C. C., Chen, W. P., \& Magnier, E. A. 2011, APRIM, V1, 181

Piskunov, A. E., Kharchenko, N. V., Röser, S., et al. 2006, A\&A, 445, 545

Wang, P. F., Chen, W. P., Lin, C. C., et al. 2014, ApJ, 784, 57 